IUCrJ

ISSN 2052-2525

PHYSICS|FELS

Received 9 March 2018

Accepted 22 October 2018

Edited by $\mathrm{H}$. Chapman, DESY/Universität Hamburg, Germany

Keywords: serial crystallography; X-ray freeelectron lasers; XFEL; electron diffraction; diffract-then-destroy; dynamical studies; auto-indexing algorithms; Bragg peaks.

Supporting information: this article has supporting information at www.iucrj.org

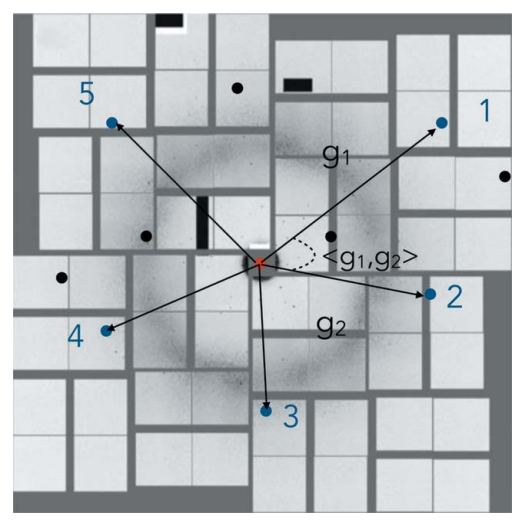

OPEN $\odot$ ACCESS

\section{SPIND: a reference-based auto-indexing algorithm for sparse serial crystallography data}

\author{
Chufeng Li, ${ }^{a, b}$ Xuanxuan Li, ${ }^{c, d}$ Richard Kirian, ${ }^{a, b}$ John C. H. Spence, ${ }^{a, b}$ Haiguang \\ Liu $^{\mathrm{c}}$ and Nadia A. Zatsepin ${ }^{\mathrm{a}, \mathrm{b}_{*}}$
}

\begin{abstract}
${ }^{\mathbf{a}}$ Department of Physics, Arizona State University, Tempe, Arizona 85287, USA, ${ }^{\mathbf{b}}$ Center for Applied Structural Discovery, The Biodesign Institute, Arizona State University, Tempe, Arizona 85287, USA, '⿳C㇒mplex Systems Division, Beijing Computational Science Research Center, Beijing, 100193, People's Republic of China, and dDepartment of Engineering Physics, Tsinghua University, Beijing, 100086, People's Republic of China. *Correspondence e-mail:

nadia.zatsepin@asu.edu
\end{abstract}

SPIND (sparse-pattern indexing) is an auto-indexing algorithm for sparse snapshot diffraction patterns ('stills') that requires the positions of only five Bragg peaks in a single pattern, when provided with unit-cell parameters. The capability of SPIND is demonstrated for the orientation determination of sparse diffraction patterns using simulated data from microcrystals of a small inorganic molecule containing three iodines, 5-amino-2,4,6-triiodoisophthalic acid monohydrate (I3C) [Beck \& Sheldrick (2008), Acta Cryst. E64, o1286], which is challenging for commonly used indexing algorithms. SPIND, integrated with CrystFEL [White et al. (2012), J. Appl. Cryst. 45, 335-341], is then shown to improve the indexing rate and quality of merged serial femtosecond crystallography data from two membrane proteins, the human $\delta$-opioid receptor in complex with a bi-functional peptide ligand DIPP- $\mathrm{NH}_{2}$ and the NTQ chloridepumping rhodopsin (CIR). The study demonstrates the suitability of SPIND for indexing sparse inorganic crystal data with smaller unit cells, and for improving the quality of serial femtosecond protein crystallography data, significantly reducing the amount of sample and beam time required by making better use of limited data sets. SPIND is written in Python and is publicly available under the GNU General Public License from https:/github.com/LiuLab-CSRC/SPIND.

\section{Introduction}

The high brightness and femtosecond pulse duration of X-ray free-electron lasers (XFELs) enabled the serial diffractionbefore-destruction paradigm (Neutze et al., 2000), which mitigates X-ray radiation damage and allows data to be collected from weakly scattering targets. In a typical serial femtosecond crystallography (SFX) experiment, diffraction patterns are recorded from tens of thousands of microcrystals delivered sequentially across a pulsed X-ray beam [Chapman et al. (2011); see Spence (2017) for a review]. These snapshot diffraction patterns (from individual microcrystals) correspond to reciprocal-space intensity samples that lie on the surface of the Ewald sphere. Since each crystal is in a random orientation, crystal orientations must be determined before intensities can be merged in three-dimensional reciprocal space. Femtosecond XFEL pulses are too short for substantial crystal rotation during exposure, so only partial reflection intensities are recorded in each diffraction pattern, with partiality determined by various factors such as X-ray bandwidth and crystal shape, size, orientation and mosaicity.

SFX data analysis is challenging because of the wide variation in crystal size and mosaicity, which is confounded by 
jitter in the XFEL pulse energy and spectrum, detector dynamic range limitations, and the random positions/orientations of crystals. Monte Carlo integration (Kirian et al., 2010) is an effective means of producing crystallographic structure factors by simply averaging over stochastic measurement variations that are assumed to be completely random. However, this approach requires a large number of patterns (on the order of tens of thousands) in order for the average partial reflection measurements to converge to a reliable set of integrated Bragg intensities [see Li et al. (2015) for error metric analysis of the Monte Carlo integration approach]. This is in contrast to conventional synchrotron crystallography in which the molecular structure is determined using one or a few larger crystals, using the oscillation approach where the crystals are rotated through the Bragg condition during the intensity recording to yield angle-integrated structure factors. Post-refinement techniques for SFX data have recently been developed that can greatly reduce the number of required snapshot patterns to a few thousand, or a few hundred, in favorable circumstances (White, 2014; Uervirojnangkoorn $e t$ al., 2015; Sauter, 2015; Ginn et al., 2015). This reduces demand on scarce XFEL beam time and sample volume.

A number of software packages have been developed specifically for SFX or snapshot diffraction data analysis [see Liu \& Spence (2016) for a review]. An SFX data-analysis pipeline commonly starts with data-reduction programs such as Cheetah (Barty et al., 2014), which apply various detector calibrations, identify diffraction peaks and produce datacollection statistics. Patterns containing diffraction peaks are then passed to programs such as CrystFEL (White et al., 2012) or cctbx.xfel (Hattne et al., 2014) for high-throughput autoindexing and intensity merging. CrystFEL's program indexamajig calls subroutines wherein partial reflections are auto-indexed and locally integrated within each two-dimensional pattern. Finally, intensities from partial reflections are merged by process_h $k l$ (optionally including scaling and postrefinement using partialator), which results in a set of Bragg intensities. For each diffraction pattern, indexamajig passes the peak positions as input arguments to auto-indexers such as MOSFLM (Powell, 1999), DirAx (Duisenberg, 1992), or XDS (Kabsch, 1988, 1993), or algorithms implemented directly in indexamajig, such as asdf, felix (Beyerlein et al., 2017) and taketwo (Ginn et al., 2016). Auto-indexing modules return a set of lattice vectors oriented in the laboratory frame and work by first converting Bragg reflections to three-dimensional reciprocal-space vectors. MOSFLM or LABELIT, for example, then projects these vectors onto a set of discrete directions distributed about a hemisphere (Campbell, 1998; Leslie, 2006; Powell, 1999; Steller et al., 1997; Sauter et al., 2004). If a sufficient number of diffraction peaks from a single crystal lattice contribute to the one-dimensional projected histogram, its Fourier transform consists of sharp spikes if the projection direction coincides with one of the principal axes of the crystal. The frequency of these spikes provides lattice parameter information. Once principal axis candidates are identified, indexamajig predicts the possible Bragg peak positions in the original pattern, tests for reasonable agreement with the observed peak positions, and if the agreement is satisfactory, the peak intensities are integrated. The result of this procedure is a set of partially integrated reflection intensities and associated Miller indices.

This data-analysis pipeline has been used for highresolution structure determination in both SFX and synchrotron serial crystallography (Nogly et al., 2016; Standfuss \& Spence, 2017). However, a significant portion of SFX diffraction patterns, especially those from difficult-to-crystallize macromolecules such as membrane proteins, only have a small number of identifiable Bragg peaks because of small crystal size, crystal disorder, scattering from air and sampledelivery medium, and detector noise, which decrease the signal-to-noise ratio (SNR). Time-resolved SFX requires small crystals because the extinction length of the optical-pump laser is typically on the order of $10 \mu \mathrm{m}$, while the temporal resolution of mix-and-inject SFX (to study ligand binding, for example) is limited by mixing and diffusion rates, and hence also benefits from smaller crystal size (Schmidt, 2013). The narrow (though spiky) bandwidth of XFELs based on selfamplified spontaneous emission (SASE) and the stillness of crystals during the ultrafast exposures in SFX also limits the number of Bragg spots that intersect the Ewald sphere. In addition, inorganic crystals typically have very small unit cells and so give rise to sparse patterns with fewer Bragg peaks in the same resolution shell than most protein crystals. Existing auto-indexing algorithms that are based on one-dimensional Fourier transforms typically require that each pattern consist of 20 [or even more (Campbell, 1998)] accurately identified Bragg peaks to yield a reliable crystal orientation. The development of auto-indexing algorithms for sparse patterns with fewer peaks would greatly increase SFX data utilization for such challenging cases with low resolution, and for samples with very small unit cells. Here we take sparse to mean that there are few Bragg spots, rather than weak Bragg intensities.

In this paper, we present a new algorithm, SPIND (sparsepattern indexing), designed to index patterns with sparse data, achieve faster and more accurate structure-factor measurements, and reduce measurement time, sample consumption and cost. The use of angles between scattering vectors, as well as their lengths, is a strong constraint, as described in Methods. SPIND has the merit of a low false-positive rate and hence a high level of effectiveness as well as efficiency, which is demonstrated on extremely sparse patterns simulated from inorganic crystals and experimental SFX data from membrane-protein microcrystals. Two alternative autoindexing algorithms for sparse patterns have been developed recently. Maia et al. (2011) developed a compressive sensingbased auto-indexing algorithm for sparse diffraction patterns in serial femtosecond nanocrystallography in which lattice reconstruction is reformulated as an L1 minimization (basis pursuit) problem. The algorithm was shown to efficiently reconstruct a three-dimensional lattice and its orientation from a simulated noise-free sparse diffraction pattern without prior knowledge of the unit cell. The use of multiple threedimensional fast Fourier transforms renders the algorithm computationally expensive in its current form, but incorpor- 
ating additional algorithms designed for sparse data should substantially increase its speed. Additionally, the indexing ambiguity arising from mirror symmetries of the lattice remains to be resolved, and the algorithm is yet to be demonstrated on experimental data or in the presence of noise. An alternative auto-indexing algorithm for sparse SFX diffraction patterns from crystals with small unit cells, which depends on known lattice parameters, was demonstrated by Brewster et al. (2015) on amyloid peptide nanocrystal data. The approach consists of three steps: (1) assign each peak all possible Miller indices corresponding to its resolution, (2) resolve the ambiguities in Miller-index assignment (e.g. from lattice symmetry or semi-overlapping powder rings) and (3) calculate basis vectors and refine crystal orientation, iteratively. The Bron-Kerbosch algorithm (Cazals \& Karande, 2008 ) is used to determine the maximum-clique of a graph in which all found peaks, with each of their candidate Miller indices, are represented as individual nodes. For each pair of peaks, the differences between calculated and observed interpeak distances in reciprocal space (for each candidate Miller index) are represented as edges, so Bragg peaks that cannot be simultaneously accounted for by one orientation matrix are not connected in the graph. An advantage of the graphic maximum-clique algorithm is its tolerance to false peaks, but for very sparse patterns (five Bragg peaks), the determined unit-cell accuracy is on the order of $10 \%$.

\section{Methods}

Here we describe the algorithm for indexing patterns containing very few Bragg peaks, which we refer to as SPIND. If peaks are sparsely distributed in each pattern, their periodicity is difficult to identify via Fourier methods. The proposed algorithm therefore utilizes prior knowledge of unitcell parameters. The algorithm works as follows (see Fig. 1 for a flowchart). For each pattern:

(a) Bragg peaks are identified and their positions, intensities, SNRs, resolution and the camera length are written to a plain text file.

(b) The two-dimensional peak positions on the detector are converted to three-dimensional reciprocal vectors that start from the beam center (treated as a 000 reflection) using the experimental geometry.

(c) The best five peaks (or more) are chosen from the peak list according to intensity, SNR or resolution (these can be chosen by the user). In principle, any number of peak pairs greater than two can be used to determine the crystal orientations. Indexing reliability increases with the number of pairs used, as discussed later. However, the computation time scales roughly as $N^{2}$ \{the number of pairs scales as $N ! /[2(N-2) !]$ where $N$ is the number of peaks\}. We found that five peaks (ten peak pairs) is a good compromise between indexing yield and computational time.

(d) For each of the ten pairs, the two vector lengths and angle between the pair are calculated [see Fig. 2(a)].

(e) For a large set of discretized crystal orientations, a reference table is created that contains the expected lengths, ratio of the lengths, and angles between vectors for all possible pairs (within a certain resolution limit). This reference table is based on prior knowledge of the unit cell and is calculated only once in the whole process of indexing.

$(f)$ The set of observed vector lengths, ratios of lengths (in case the geometry needs refinement), and angles corresponding to each of the ten pairs of peaks are compared with the entries in the reference table. Whenever a match is found between a given observed peak pair and an entry in the reference table (within a preset mismatch tolerance), the reference-table entry (i.e. crystal orientation) is considered to be a solution candidate and added to a solution pool. One solution pool, with multiple entries, is created for each of the ten peak pairs.

$(g)$ If all reciprocal vectors correspond to the same crystal orientation, the true solution for the crystal orientation must be in the intersection of all ten solution pools (Fig. 2b). This is a strong constraint that effectively eliminates spurious solution candidates in one step without implementing other optimization and clustering algorithms such as in the work by Brewster et al. (2015). However, this constraint is sensitive to peaks not belonging to a single lattice, which will require development of approaches for eliminating false peaks used for indexing. The indexing ambiguity that occurs in cases where the symmetry of Bravais lattice is higher than the spacegroup symmetry is not resolved here. Several algorithms have been designed and tested to resolve this type of indexingambiguity problem, in particular for serial crystallography data (Brehm \& Diederichs, 2014; Liu \& Spence, 2014).

(h) For very sparse patterns, if a single orientation is found in the intersection, it is used to predict peak locations. If the distance between predicted and found peaks of the same Miller index (in three-dimensional reciprocal space) is within the tolerance threshold, the peaks are tagged as matched. By default, this threshold is half of the distance to the nearest Bragg peak. If the predicted peaks match all found peaks in the experimental pattern, the solution is accepted; otherwise, the pattern is considered un-indexable and rejected.

(i) For diffraction patterns containing more spots: all candidate orientations (from all solution pools) are used to predict peak locations. The quality of the orientation candidate solution is scored by the number of predicted peaks matching observed peaks (for all peaks, not just those used for indexing), the percentage of peaks matched (a higher percentage of matched peaks is required if the total number of peaks is low) and the determined lattice centering.

\section{Results and discussions}

3.1. Simulating and indexing sparse patterns from inorganic microcrystals

In order to test the ability of SPIND to index sparse diffraction patterns, we simulated 400 diffraction patterns from 5-amino-2,4,6-triiodoisophthalic acid monohydrate (I3C) crystals (Beck \& Sheldrick, 2008) at random orientations. A 

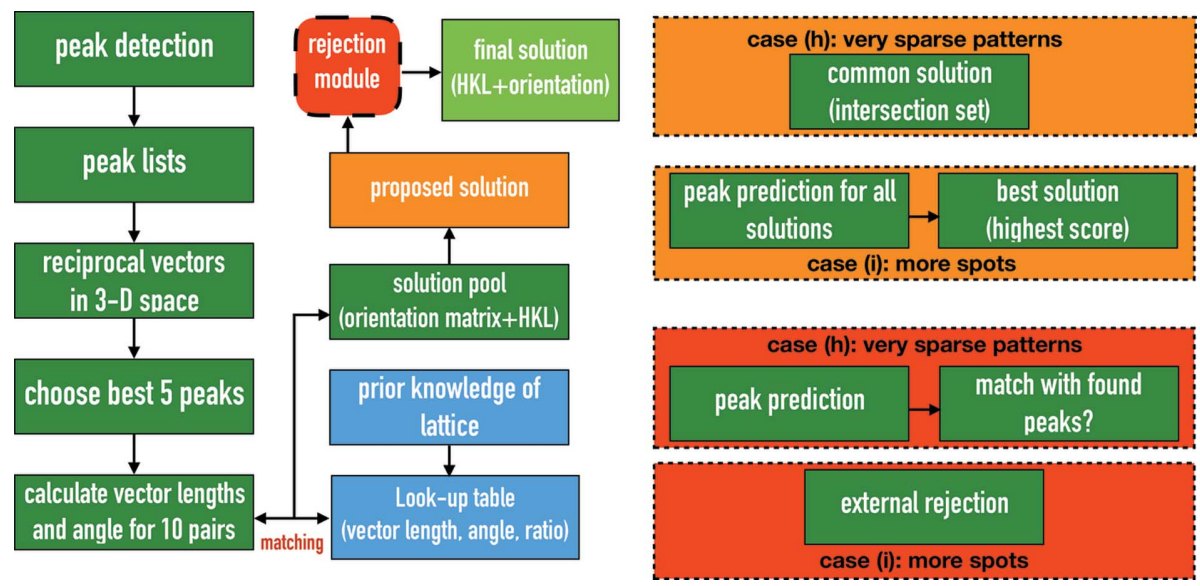

Figure 1

Flowchart of the SPIND indexing algorithm. The five best peaks in each pattern selected based on user-chosen criteria, such as SNR, are used for indexing. The blue boxes refer to prior knowledge and the table is calculated once. The green boxes are steps carried out for each pattern. The (red) rejection module refers to steps $(g)$ to $(i)$.
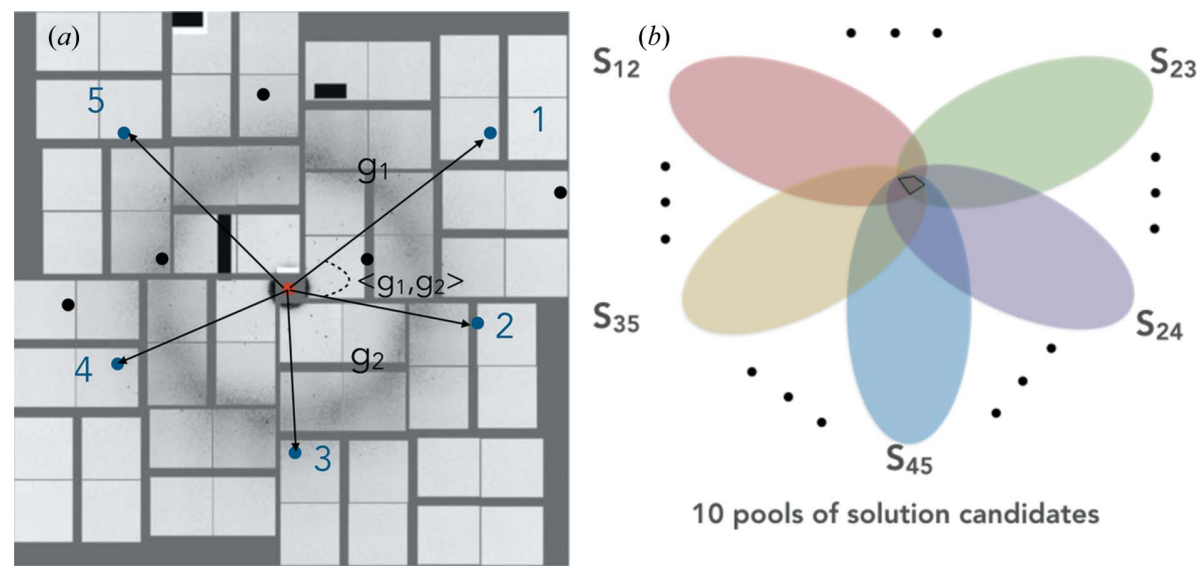

10 pools of solution candidates

Figure 2

Illustration for SPIND auto-indexing algorithm. (a) A diffraction pattern recorded by Cornell-SLAC hybrid Pixel Array Detector (CSPAD) (Herrmann et al., 2013; Hart et al., 2012), with a few exaggerated peaks for illustrative purposes. Five peaks are selected to form ten vector pairs. The vector lengths, ratio of lengths and angles between the vectors are then calculated for the ten pairs for matching with a reference based on a priori knowledge of the unit cell (within some mismatch tolerance). (b) Rejection module for eliminating spurious solution candidates, based on the constraint that all peak pairs share the same crystal orientation. The solution must lie in the intersection of the solution pools, provided that the peaks are from a single crystal.

unit cell with $a=9.02, b=15.73, c=18.82 \AA, \alpha=\beta=\gamma=90^{\circ}$, a photon energy of $9.61 \mathrm{keV}, 0.5 \mu \mathrm{m}$ beam radius, $110 \times$ $110 \mu \mathrm{m}^{2}$ detector pixel size, and $53.2^{\circ}$ maximum scattering angle at a working distance of $0.07 \mathrm{~m}$ were used for diffraction-pattern simulations. The three-dimensional profile of the reciprocal lattice points was modeled as Gaussians taking into account $1 \mu \mathrm{m}$ crystal size and structure factors. The intensities were calculated from the three-dimensional Gaussian profile and the excitation error, defined as the distance between the Ewald sphere (corresponding to monochromatic X-ray beam of $9.61 \mathrm{keV}$ photon energy) and the center of the reciprocal lattice point projected in the beam direction. Poisson noise and background scattering were included such that only three to five Bragg peaks were identifiable in each pattern [see Figs. 3(a) and 3(b) for a representative pattern before and after adding noise terms]. The SPIND algorithm obtained correct crystal orientations and Miller indices for all 400 patterns. This implementation of SPIND (written in MATLAB R2014b; The MathWorks Inc., Natick, MA, USA) required millisecond computational time on a Mac $2.7 \mathrm{GHz}$ Intel Core i7. In addition to the fast computation time, the orientation was determined with high accuracy. A typical example from the 400 patterns that were indexed successfully is shown in Fig. 3(c), where the orientation was determined with an accuracy of around $0.1^{\circ}$.

To investigate the robustness of the indexing algorithm in the presence of lattice inhomogeneity or inaccurate guiding-cell constants, an additional set of 400 I3C diffraction patterns was simulated with random Gaussian fluctuations in lattice constants about the mean values with $0.5 \%$ standard deviation. SPIND indexing was carried out using 11 different guiding unit cells with varying $\alpha$ angle values and lengths of $b$ and $c$ basis vectors. The $\alpha$ angle of the guiding cell was varied from 80 to $110^{\circ}$ symmetrically around the nominal value of $90^{\circ}$, with the length of the $b$ and $c$ basis vectors adapted such that the volume of the cell is invariant (same as the nominal cell used to simulate the diffraction patterns) to maintain a consistent average density of the Bragg orders as a control factor. The number of indexed patterns decreased as the guiding cell deviated incrementally from the nominal cell (Fig. $4 a$ ). In this test, the peak indexing rate appears at the $\alpha$ angle values of 89 and $91^{\circ}$, which is most likely attributable to the random fluctuations in lattice constants combined with the limited size of the diffraction data set. To validate this argument, the distribution of the three-dimensional reciprocal-space distances between the predicted and the found positions for the paired peaks from all indexed patterns were obtained for the $\alpha$ angle values of 90, 91, 92 and $93^{\circ}$ (Fig. $4 b$ ). The most probable value of the distance between the predicted and the observed peak positions is minimized when $\alpha=90^{\circ}$ and increases consistently as the $\alpha$ value incrementally deviates from $90^{\circ}$. In addition, the total number of paired peaks also drops as the guiding cell deviates from the nominal. In accordance with the symmetry of the orthorhombic I3C lattice, the indexing rate and distance discrepancy for paired peaks are also essentially symmetric about $\alpha=90^{\circ}$. 


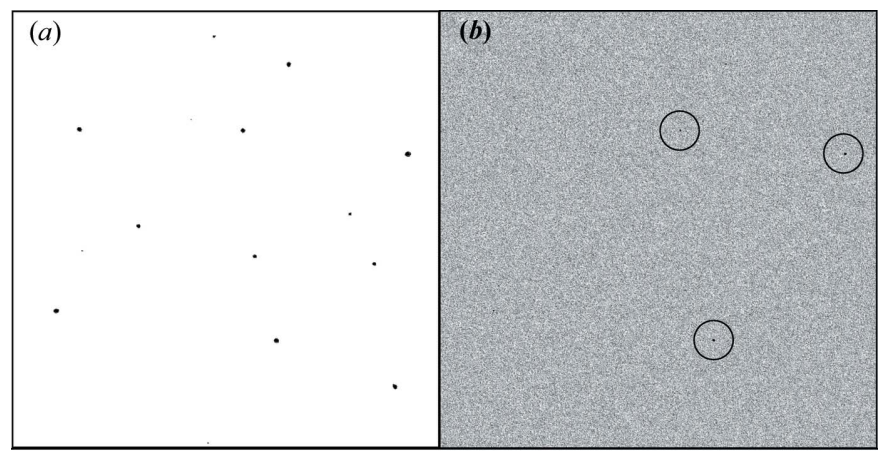

(c)

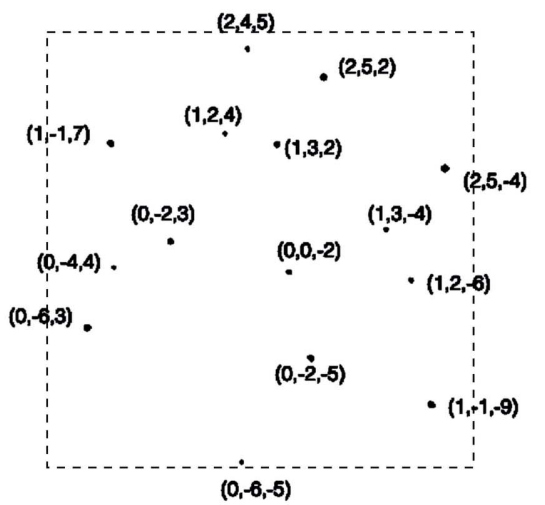

Figure 3

Simulated I3C patterns indexed by SPIND. (a) Simulated sparse diffraction pattern from an I3C crystal in the orientation specified by Euler angles -10.4676, 46.9022, 139.1443. (b) Poisson noise and random background noise added to $(a)$, so only three Bragg peaks were identifiable (circled). (c) The indexing result by SPIND using only the three peaks in $(b)$. The determined crystal orientation is at Euler angles of $-10.5713,46.8855,139.2000$. The peaks were predicted from the determined orientation and Miller indices were given for Bragg peaks.
For this reason, only half of the distribution statistics, corresponding to $\alpha>90^{\circ}$, are shown in Fig. 4(b) for visual clarity. The abrupt drops of the paired-peak population at $0.01 \AA^{-1}$ in Fig. 4(b) arise from this same cut-off value set for the peakmatch judgment [step $(h)$ in the Methods section]. The indexing rate drops to approximately $25 \%$ at $\alpha=87,93^{\circ}$ which shows a $\pm 3^{\circ}$ tolerance range for the uncertainty or inaccuracy in the guiding-cell constants. The indexing rate drops rapidly further to $0.5 \%$ at $\alpha=85,95^{\circ}$ and goes to 0 beyond $10^{\circ}$ of deviation. This low false-positive indexing rate verifies the reliability of the SPIND rejection module. These indexing statistics using guiding cells that deviate incrementally from the nominal have demonstrated the robustness of the SPIND indexing algorithm to the lattice inhomogeneity, a wide tolerance range for the guiding-cell constants and low falsepositive indexing rate when the target lattice cell is clearly distinguishable from the guiding cell. Furthermore, it is worthwhile to point out that the guiding cell, as well as other indexing parameters such as error tolerance and threshold values (see the Methods section), can be updated iteratively by using indexing statistics (see Fig. 4 as an example) as feedback. Individual pattern-based lattice refinement, together with this iterative updating of the guiding cell and indexing parameters are part of ongoing development.

\subsection{Indexing SFX data from a G-protein-coupled receptor complex}

To demonstrate the algorithm on protein serial crystallography data, SPIND was used to index serial X-ray diffraction data from microcrystals of a G-protein-coupled

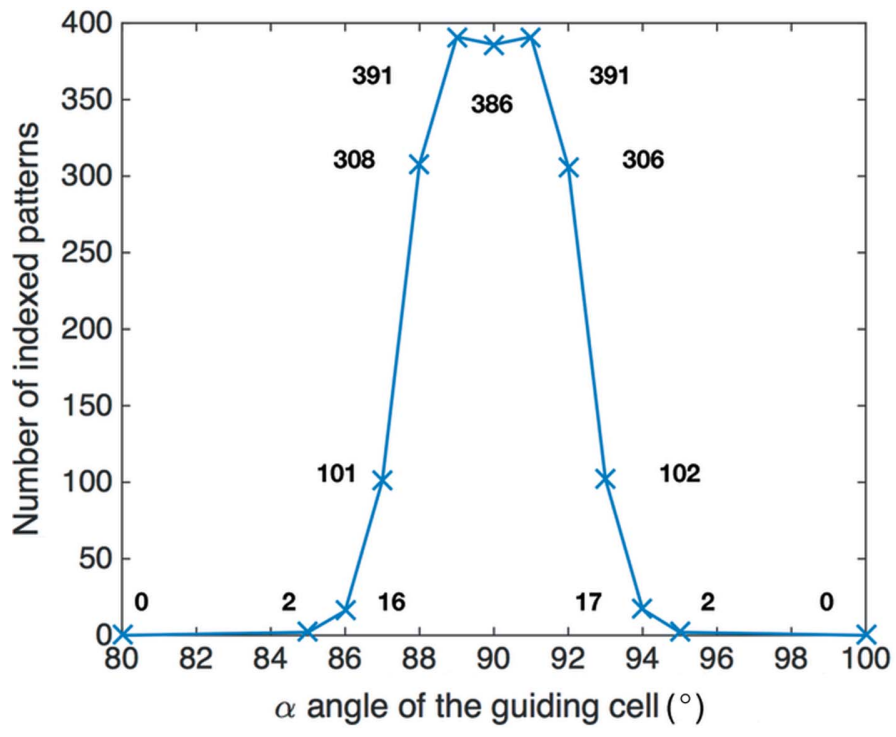

(a)

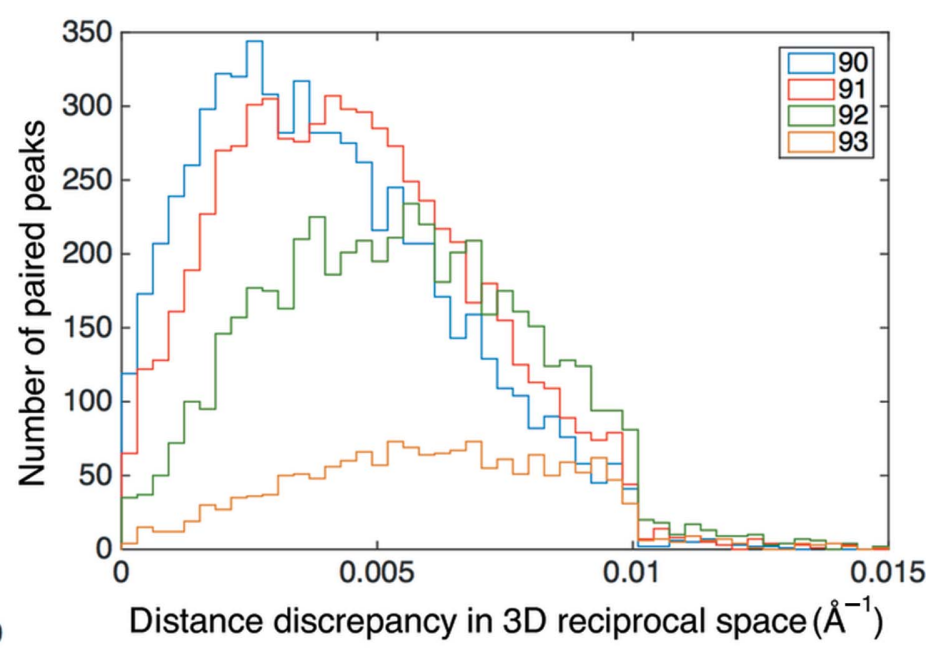

(b)

Figure 4

The effect of inaccurate guiding unit cells on SPIND indexing rates and peak-prediction accuracy demonstrated on simulated I3C snapshot diffraction patterns. (a) Number of indexed patterns as a function of the $\alpha$ angle of the guiding cell $\left(\alpha=90^{\circ}\right.$ is nominal). (b) Distribution of distance discrepancy in three-dimensional reciprocal space between found and predicted peaks for matched peak pairs using guiding cells with different $\alpha$ angle values. The legend shows the $\alpha$ angle of the guiding cell. The center of the distribution shifts to larger values as $\alpha$ deviates further from the nominal value of $90^{\circ}$. The same trend was observed for values of $\alpha<90^{\circ}$ (omitted for clarity). The results demonstrate the robustness of the algorithm to the lattice inhomogeneity, a wide tolerance range for the guiding-cell constants and low false-positive indexing rate when the target lattice cell is clearly distinguishable from the guiding cell. The indexing rate can be used as an indicator for the accuracy of the reference unit cell. 
Table 1

Parameters for hit finding of DOR data set using Cheetah (Fenalti et al., 2015).

\begin{tabular}{llllllll}
\hline $\begin{array}{l}\text { No. of } \\
\text { frames } \\
\text { collected }\end{array}$ & $\begin{array}{l}\text { Minimum no. } \\
\text { of peaks } \\
\text { per frame }\end{array}$ & $\begin{array}{l}\text { No. of } \\
\text { pixels } \\
\text { per peak }\end{array}$ & $\begin{array}{l}\text { Peak } \\
\text { intensity } \\
\text { threshold } \\
\text { (ADU) }\end{array}$ & $\begin{array}{l}\text { SNR } \\
\text { threshold }\end{array}$ & $\begin{array}{l}\text { Peak } \\
\text { search } \\
\text { region }\end{array}$ & $\begin{array}{l}\text { Peak } \\
\text { finder } \\
\text { algorithm }\end{array}$ & $\begin{array}{l}\text { No. of } \\
\text { hits found } \\
\text { (hit rate \%) }\end{array}$ \\
\hline 1967539 & 15 & $2-40$ & 40 & 4 & $\begin{array}{l}70-700 \text { pixels } \\
\text { from center }\end{array}$ & 8 & $125458(5.9)$ \\
\hline
\end{tabular}

Table 2

Parameters for auto-indexing of DOR SFX data using indexamajig, calling DirAx, MOSFLM and SPIND as subroutines.

\begin{tabular}{|c|c|c|c|c|c|}
\hline Indexer & $\begin{array}{l}\text { Reference } \\
\text { cell }\end{array}$ & $\begin{array}{l}\text { indexamajig } \\
\text { orientation } \\
\text { refinement }\end{array}$ & $\begin{array}{l}\text { Unit-cell tolerance } \\
\text { (vector-search } \\
\text { tolerance } \\
\text { in } S P I N D \text { ) }\end{array}$ & $\begin{array}{l}\text { Radii of } \\
\text { peak integration } \\
\text { and background } \\
\text { rings (pixels) }\end{array}$ & $\begin{array}{l}\text { No. of } \\
\text { indexed patterns } \\
\text { (indexing rate \%) }\end{array}$ \\
\hline $\operatorname{Dir} A x$ & Yes & Yes & $5 \%, 1.5^{\circ}$ & $3,4,5$ & $65015(51.8)$ \\
\hline MOSFLM & Yes & Yes & $5 \%, 1.5^{\circ}$ & $3,4,5$ & $57687(45.9)$ \\
\hline MOSFLM & No & Yes & $5 \%, 1.5^{\circ}$ & $3,4,5$ & $57413(45.7)$ \\
\hline SPIND & Yes & Yes & $\begin{array}{l}5 \%, 1.5^{\circ} \\
\quad\left(3 \times 10^{7} \mathrm{~m}^{-1},\right. \\
3^{\circ}, \text { reference } \\
\text { resolution }>8 \AA)\end{array}$ & $3,4,5$ & $67204(53.5)$ \\
\hline SPIND & Yes & No & $\begin{array}{l}5 \%, 1.5^{\circ} \\
\quad\left(3 \times 10^{7} \mathrm{~m}^{-1},\right. \\
3^{\circ}, \text { reference } \\
\text { resolution }>8 \AA)\end{array}$ & $3,4,5$ & $118514(94.4)$ \\
\hline
\end{tabular}

used for the original processing (Fenalti et al., 2015). The indexing was attempted by MOSFLM 7.2.0 (using prior unit-cell parameters and lattice type information), followed by $\operatorname{Dir} A x$, and finally MOSFLM without any prior information. For a fair comparison of the indexing rate and accuracy of SPIND on SFX data, the DOR data were reprocessed using one indexing method at a time rather than combinations thereof [the latter was the approach in the work by Fenalti et al. (2015) and (White, Barty et al., 2016). The auto-indexing methods that are compared in this work are SPIND, DirAx, and MOSFLM (with and without lattice type and unit-cell input). Also, the refinement option was toggled on and off in indexamajig to investigate the effect of the pattern filtering after SPIND auto-indexing on the quality of merged data.

The data processing was performed using the scripts deposited in CXIDB ID 40 with minimal

Table 3

Merging statistics from DOR SFX data using partialator.

The values in the parentheses are for the highest-resolution shell (2.8-2.7 $\AA$ )

\begin{tabular}{|c|c|c|c|c|c|c|}
\hline $\begin{array}{l}\text { Indexing } \\
\text { method }\end{array}$ & $\begin{array}{l}\text { No. of } \\
\text { patterns } \\
\text { used }\end{array}$ & $\begin{array}{l}\text { No. of } \\
\text { crystals } \\
\text { merged }\end{array}$ & $\begin{array}{l}\text { Resolution } \\
\text { range }(\AA)\end{array}$ & $\begin{array}{l}R_{\text {split }} \\
(\%)\end{array}$ & $\mathrm{CC}^{*}$ & SNR \\
\hline $\begin{array}{l}\operatorname{Dir} A x \\
\quad \text {-refine }\end{array}$ & 65015 & 64918 & $34.3-2.7$ & 11.9 & $\begin{array}{l}0.9982 \\
\quad(0.7069)\end{array}$ & 6.3 \\
\hline $\begin{array}{c}M O S F L M \\
\text {-refine }\end{array}$ & 57687 & 57597 & $34.3-2.7$ & 12.6 & $\begin{array}{l}0.9979 \\
\quad(0.6803)\end{array}$ & 6.1 \\
\hline $\begin{array}{c}\text { MOSFLM } \\
\text {-nolatt } \\
\text {-refine }\end{array}$ & 57413 & 57309 & $34.3-2.7$ & 12.1 & $\begin{array}{l}0.9980 \\
\quad(0.6704)\end{array}$ & 6.2 \\
\hline $\begin{array}{l}\text { SPIND } \\
\quad \text {-refine }\end{array}$ & 67204 & 67067 & $34.3-2.7$ & 11.7 & $\begin{array}{l}0.9981 \\
\quad(0.6992)\end{array}$ & 6.5 \\
\hline $\begin{array}{l}\text { SPIND } \\
\quad \text {-norefine }\end{array}$ & 118514 & 116198 & $34.3-2.7$ & 14.7 & $\begin{array}{l}0.9973 \\
\quad(0.5978)\end{array}$ & 5.8 \\
\hline
\end{tabular}

receptor (GPCR) complex, the human $\delta$-opioid receptor in complex with a bi-functional peptide ligand DIPP-NH2 (referred to as DOR henceforth) collected at the Coherent X-ray Imaging (CXI) endstation of the Linac Coherent Light Source (LCLS) (Fenalti et al., 2015; Liang et al., 2015).

We used the diffraction patterns from the data set ID 40 in the Coherent X-ray Imaging Data Bank [CXIDB, Maia (2012)]. For CXIDB 40, the LCLS raw data, containing over 1967530 detector frames, were reduced using Cheetah for hit finding, leaving 125458 diffraction patterns from DOR microcrystals. Indexing and intensity integration were performed with CrystFEL 0.6.2 (White, Barty et al., 2016), based on the same indexamajig and partialator parameters as necessary changes to keep the consistency for comparison between different indexing methods. A slightly larger unit cell than the published one was found to give more symmetric unit-cell distributions (see Fig. S1 in the Supporting information) and higher indexing rates, so the updated unit cell was used for all DOR indexing tests, with $a=160.10, b=91.64, c=$ $99.05 \AA, \beta=92.22^{\circ}$. The other parameters used for data processing in this work are summarized in Tables 1 and 2. In general, the number of indexed patterns using SPIND algorithm increases with the resolution limit that the reference table is generated to [step (e) in the Methods]. However, increasing the resolution limit of the reference table also results in longer computing time and higher demands on memory. Therefore, an optimal resolution limit of $8 \AA$ was chosen after several trials to balance between the indexing rate and the computation time. Similarly, the tolerance threshold for reciprocal-vector search [step $(f)$ ] was set to be $3 \times 10^{7} \mathrm{~m}^{-1}$ and $3^{\circ}$ based on the performance of several trials.

To evaluate the performance of SPIND alongside other indexing methods, the merged data quality metrics - SNR, multiplicity, and two metrics of data precision: CC* (Karplus $\&$ Diederichs, 2012) and $R_{\text {split }}$ (White, Kirian et al., 2012) - are summarized in Table 3 and plotted in Fig. 5. Compared with MOSFLM or DirAx individually, SPIND yielded the highest indexing rate $(53.5 \%)$ with the orientation refinement option on in indexamajig (see Table 3), and $94.4 \%$ without the refinement. The refinement module in indexamajig of CrystFEL acts as a filter for the indexed patterns (White, Mariani et al., 2016) by conserving only the patterns where the predicted peaks match the observed peaks sufficiently well 
while discarding the others, resulting in a reduced indexing rate. The multiplicity from DirAx-refine is slightly higher than that from SPIND-refine, while the overall SNR is lower (Fig. 5). SPIND-refine has the highest overall figures-of-merit - SNR, $R_{\text {split }}$, and CC ${ }^{*}$ (Karplus \& Diederichs, 2012) - in all resolution shells. These self-consistency figures-of-merit along with the Wilson plots (Fig. 6) for the merged data sets from different indexing methods verify the general reliability of the SPIND indexing algorithm and its applicability to serial protein crystallography data processing. (Otherwise, the figures-of-merit would become worse as patterns that are incorrectly indexed are merged towards the structure-factor list.) The $B$ factors [calculated by TRUNCATE in CCP4 (French \& Wilson, 1978)] were between $48 \AA^{2}$ (MOSFLMnolatt-refine) and $60 \AA^{2}$ (SPIND-norefine). Moreover, the

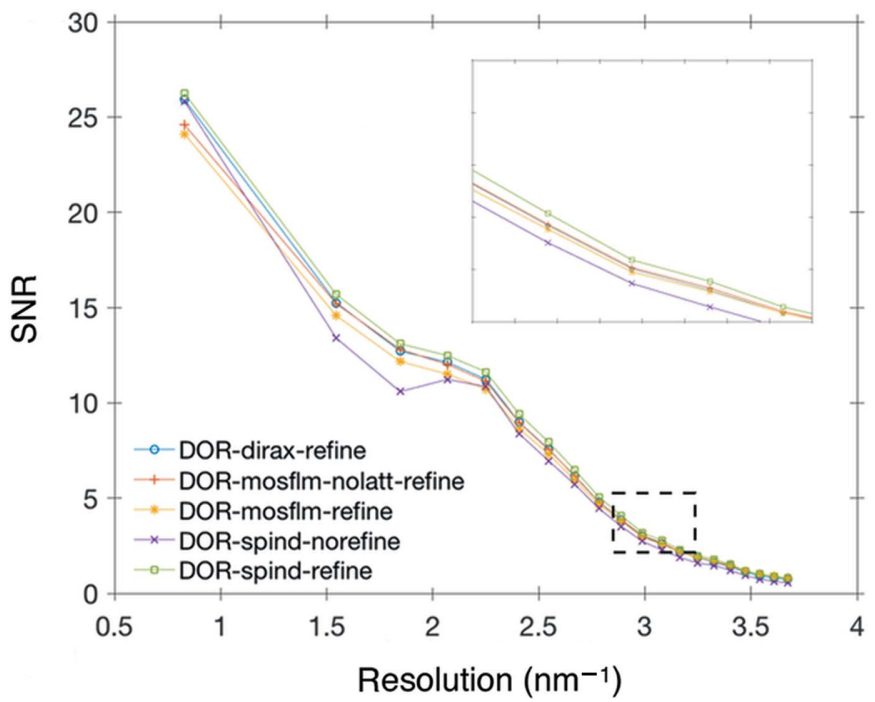

(a)

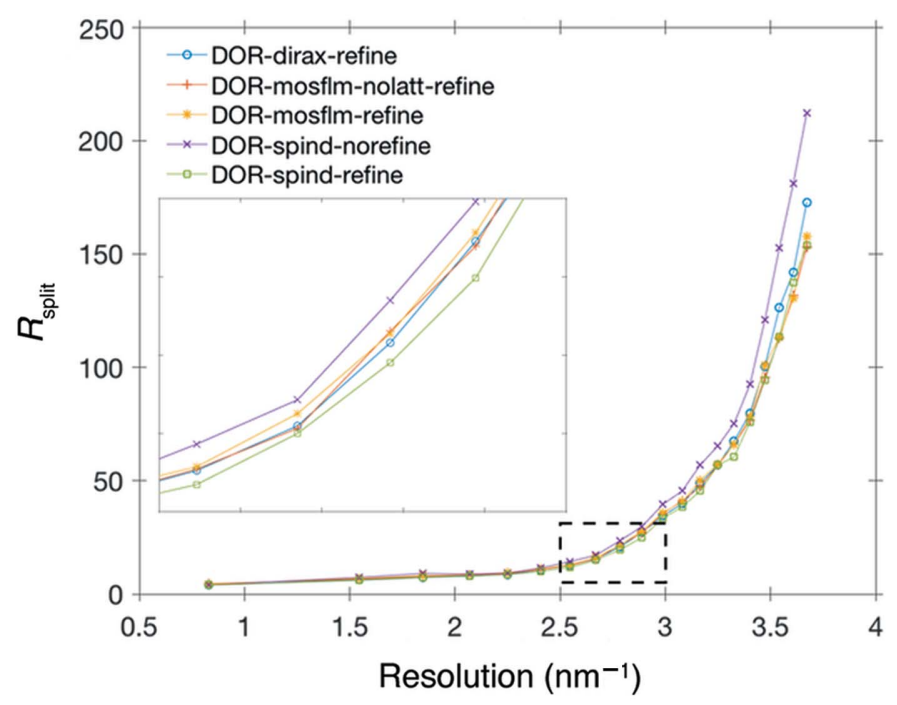

(c) additional patterns indexed using SPIND and the improved figures-of-merit indicate the potential capability of mining more data for structure determination, and hence improving the data efficiency, by including this new algorithm in the serial crystallography data analysis routine.

By enabling indexing of patterns with much fewer peaks, SPIND-norefine yields much higher multiplicity [see Fig. S2(b) in the Supporting information], at the cost of other figures-of-merit (Fig. 5). The Bragg reflection profile radius (for each crystal) calculated by indexamajig is a measure of the excitation errors of matched peaks and thus serves as a measure of the accuracy of the determined orientation. The modal value of the reflection profile radii when using SPINDnorefine is the same as for the other indexing methods (Fig. $5 d$ ), indicating SPIND orientation determination is

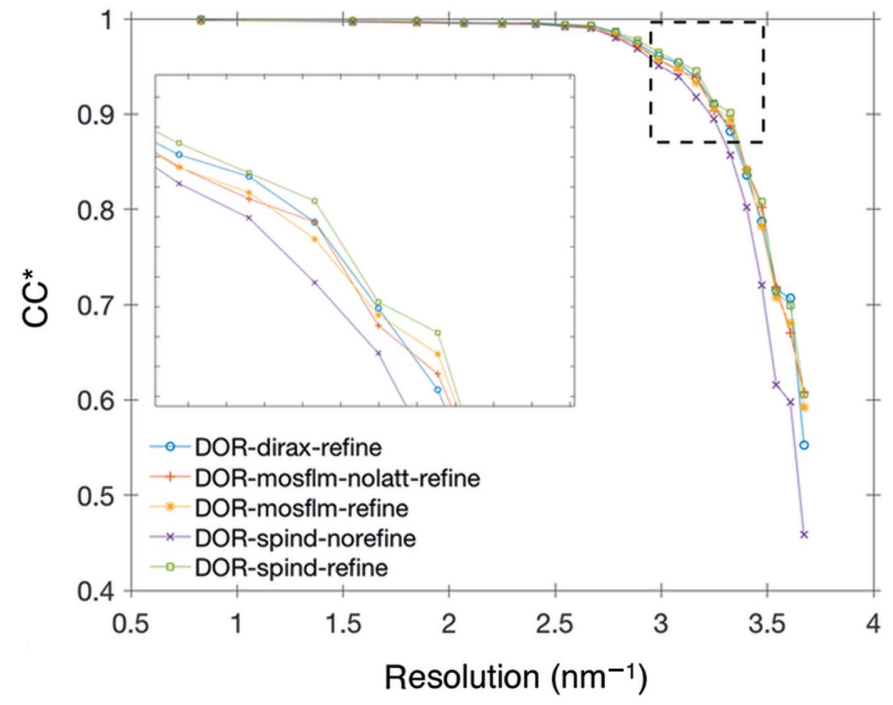

(b)

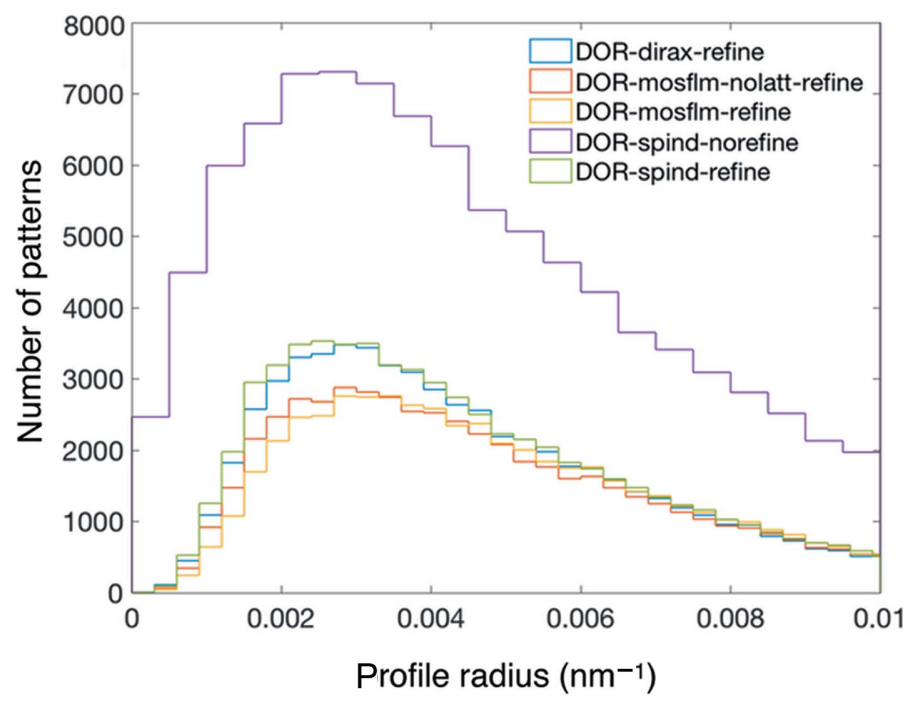

$(d)$

Figure 5

Figures-of-merit as a function of resolution for DOR SFX data. (a) SNR, $(b) \mathrm{CC}^{*},(c) R_{\text {split }}$ and $(d)$ Bragg reflection profile radii determined by indexamajig. See Fig. S2(a) for full range of reflection profile radii and Fig. S2(b) for reflection multiplicity in merged data sets in the Supporting information. The keywords 'refine' and 'norefine' represent the on and off status of the lattice-refinement option in indexamajig in the indexing process. 'nolatt' represents that the reference cell and lattice type were not used as input for indexing (but were used as constraints for the indexing solution). 


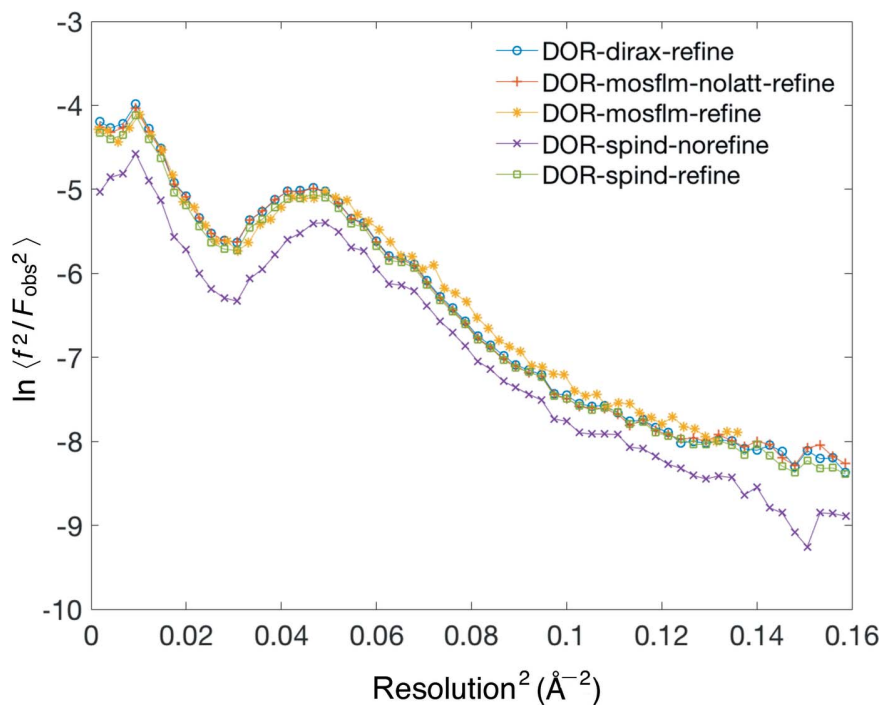

Figure 6

Wilson plots for the merged DOR data sets from different indexing methods. The linearity in the 0.06 to $\sim 0.13 \AA^{-2}$ region and the consistency between all indexing methods confirm the quality of the merged data sets.

sufficiently accurate for these patterns without the refinement module in indexamajig. However, $10 \%$ of the patterns had an insufficient number of matched peaks as determined by indexamajig, and the reflection profile radius was not updated from the default value of $0.02 \times 10^{9} \mathrm{~m}^{-1}$ [see Fig. S2 $(a)$ in the Supporting information]. Removal of these patterns improved the SNR (and the $\mathrm{CC}^{*}$, marginally) in lower-resolution bins. The majority of DOR patterns were of lower resolution (see Fig. S3 in the Supporting information), and SPIND-norefine was able to index a larger portion of these [see Fig. S3 $(f)$ in the Supporting information], with high accuracy indicated by the small reflection profile radii [see Fig. $\mathrm{S} 4(b)$ in the Supporting information]. It is possible the lower overall quality of the merged SPIND-norefine data set is caused by the inclusion of potentially anisomorphous crystals (from the lower-resolution crystal batch), or lack of orientation and unitcell refinement.

\subsection{Indexing SFX data from chloride ion-pumping rhodopsin microcrystals}

Serial crystallographic data were collected from chloridepumping rhodopsin (ClR) microcrystals at CXI, LCLS. Over 1200000 raw frames were collected in about $3 \mathrm{~h}$. Cheetah identified 105050 patterns as crystal hits with at least ten peaks per pattern with $\mathrm{SNR}>8$. After several rounds of geometry and lattice-cell refinement, 3414 patterns were indexed using CrystFEL, giving an indexing rate of approximately $3 \%$, with a monoclinic unit cell where $a=103.45, b=$ 50.28, $c=69.38 \AA$, and $\beta=109.7^{\circ}$. Merging these 3414 indexed patterns led to a structure solution (based on molecular replacement) but with low figures-of-merit. This was attributed to the small number of indexed diffraction patterns (especially for a membrane protein) and poor diffraction quality in the higher-resolution range (the average multiplicity drops below 15 for resolution greater than $6 \AA$ ).

To understand what caused the $3 \%$ low indexing rate, statistics including peak intensity and number of peaks per pattern were obtained for all the crystal hits. A strong correlation between the number of peaks per pattern and the indexing rate was found as shown in Fig. 7(a). For patterns that consist of more than 100 peaks, the indexing rate is above $\sim 30 \%$ (CrystFEL 0.6.2), while it drops significantly for the patterns with fewer peaks. Furthermore, as shown by the distribution of number of peaks per pattern (Fig. 7a), a large portion of the patterns that are identified as crystal hits consist of only ten to 30 peaks. This ineffectiveness and low efficiency in auto-indexing the patterns with a small number of peaks led to the low indexing rate of $3 \%$. To improve the indexing rate for patterns that consist of few peaks, the SPIND algorithm was applied to this data set. The indexing rates from all indexing methods are summarized and compared in Fig. 7(b). The SPIND algorithm increased the indexing rate slightly, to $4 \%$ if using the refinement feature in indexamajig (labeled SPIND-refine in the figures), and to $54 \%$ without the refinement feature. MOSFLM indexed $3.1 \%$ of the patterns using refinement and $3.5 \%$ without the refinement step. SPIND orientation solutions were chosen based on scoring the candidate orientations as described in the Methods [step (i)].

Lattice and orientation refinement in indexamajig requires that more than nine peaks match the predicted peak positions well, from the lowest resolution, with a smooth gradient in excitation error for later refinement. Patterns are regarded as unindexed if this criterion is not met. This contributes to the abrupt reduction in indexing rate when the refinement is included in the analysis of the data set since it consists of a significant portion of patterns with few peaks. Fig. 8(a) shows a representative diffraction pattern that is successfully indexed by both MOSFLM and SPIND. Almost all patterns indexed by $M O S F L M$ were also indexed by SPIND (with consistent crystal orientations). Patterns that MOSFLM could index but $S P I N D$ failed to index were found to be multi-crystal patterns. $S P I N D$ indexed more patterns that were not indexed using MOSFLM, and an example is shown in Fig. 8(b). It should be noted that the pattern in Fig. $8(b)$ has fewer peaks than that in Fig. 8(a). This observation is consistent with the correlation between indexing rate and number of peaks per pattern identified in Fig. 7(a), and validates the capability and effectiveness of the SPIND algorithm in indexing patterns with fewer peaks in this data set.

The CIR data sets were merged with partialator (version 0.6.3), excluding reflections with pixel values > $13200 \mathrm{ADU}$, push-res $=1.0$, with three iterations of scaling and no partiality refinement, resulting in high SNR and $\mathrm{CC}^{*}$, but limited completeness at high resolution (making the $\mathrm{CC}^{*}$ and SNR misleading in those resolution bins), as shown in Figs. 9 and S5 in the Supporting information.

The Wilson plots are linear to $\sim 2 \AA$ for all indexing methods (Fig. 9d). SPIND-refine performed the best overall, with a higher indexing rate, slightly higher SNR, CC* and smaller modal reflection profile radius (Fig. 9). The inclusion 


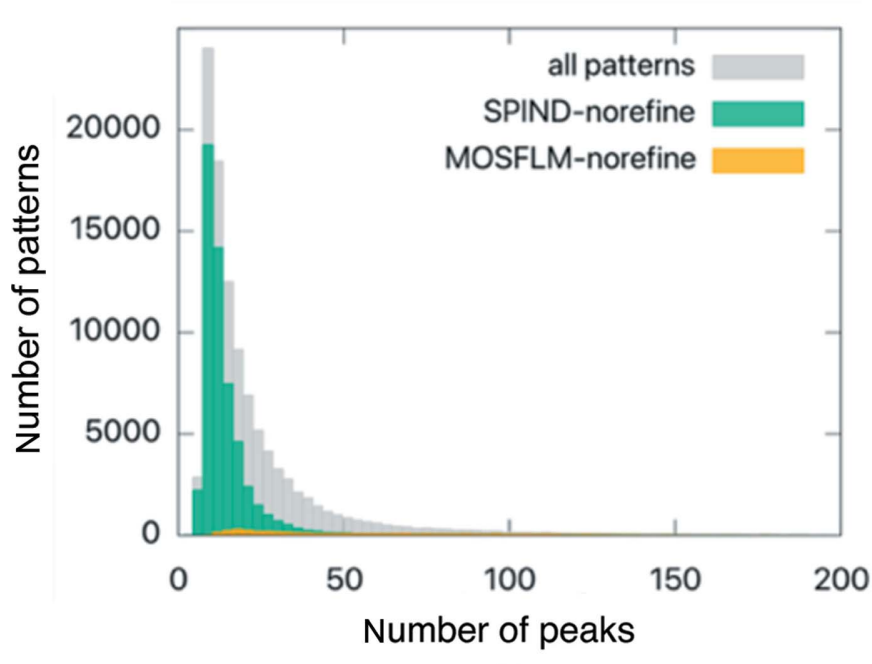

(a)

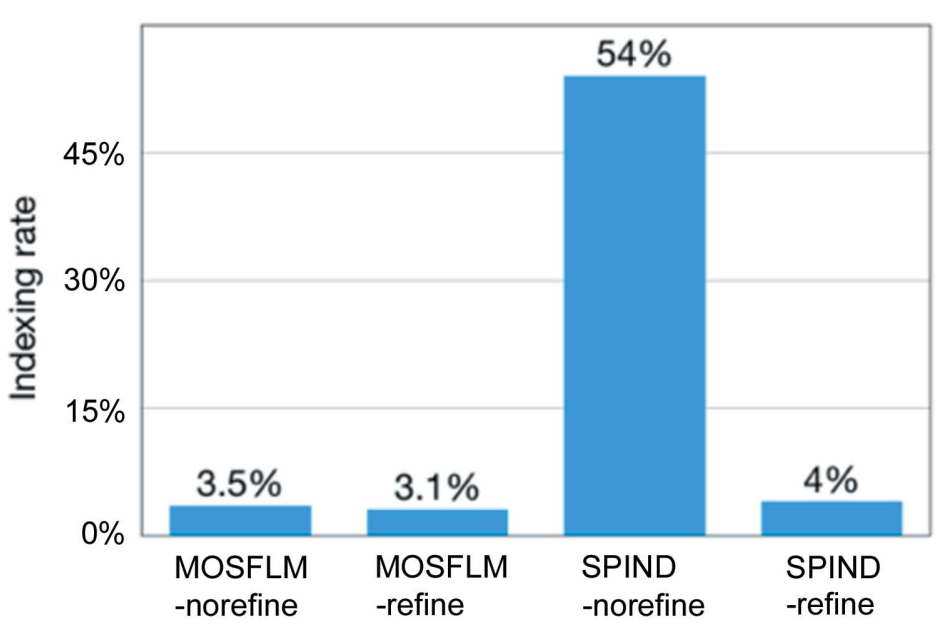

(b)

Figure 7

Statistics of ClR data set. (a) Distribution of number of peaks per pattern. Most patterns contained ten to 30 peaks, and were not indexed using MOSFLM (gray bars, 100 000 patterns). Histograms from SPIND-refine and MOSFLM-refine fit within the yellow distribution and are omitted for clarity. (b) Comparison of indexing rates using MOSFLM and SPIND with the lattice-refinement option in indexamajig enabled and disabled. The lattice-refinement feature requires that more than ten found peaks match their predicted peak positions with a small excitation error (that increases smoothly with resolution) (White, Barty et al., 2016). Patterns are discarded (not indexed) if this criterion is not met. This contributes to the abrupt cut in indexing rate from using $S P I N D$-norefine to $S P I N D$-refine since this data set consists of a significant portion of patterns with few peaks (fewer than five).

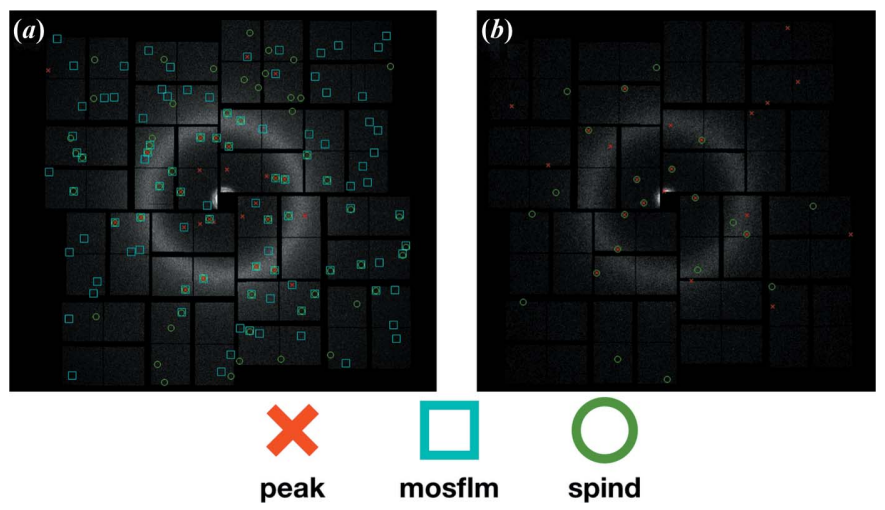

Figure 8

Representative indexed diffraction patterns from the CIR data set, recorded on the CSPAD. (a) indexable by both MOSFLM and SPIND, (b) indexable only by SPIND. Identified peaks are marked by red crosses, and peak positions predicted from the orientation matrix given by MOSFLM and SPIND are marked with cyan and green circles, respectively. The overlapping cyan and green circles in (a) correspond to the same Miller indices, thus confirming the consistency of the indexing results between SPIND and MOSFLM.

of a large number of low-resolution patterns by SPINDnorefine yielded a higher SNR and $\mathrm{CC}^{*}$ in the lowest resolution shell, but performed worse at medium and high resolution. To understand the contrasted behavior between low- and high-resolution ranges, the histograms of the apparent diffraction resolution that is estimated by CrystFEL per pattern for different indexing methods are compared with the resolution histogram of the found peaks (Fig. 10). The resolution distributions of the patterns indexed by SPIND are consistent with that of all found peaks, showing clustering in both low- and high-resolution ranges, while other indexing methods favor more high-resolution patterns. The peak in the low resolution range tails at about $1.2 \mathrm{~nm}^{-1}$ in Figs. $10(d)$ and 10(e) explains the significant increase in SNR in the lowest resolution bin (Fig. 9b). The kink in the SPIND-norefine Wilson plot (Fig. $9 d$, yellow line) around $0.15 \AA^{-2}$ is probably caused by the imperfect scaling of intensities from two distinct crystal batches, judging by their diffraction resolution (see resolution histograms in Fig. 10e), which is likely to be indicative of anisomorphism between the two crystallization batches. The accuracy or orientations determined by SPINDnorefine can be inferred by the small reflection profile radii, showing again that SPIND, even without orientation refinement, did determine accurate orientations for the majority of the patterns.

The refinement module in indexamajig optimizes and refines the lattice constants and crystal orientation for each indexed pattern by minimizing the residuals between the experimental peak positions and the peak positions that are predicted from the orientation matrix given by the autoindexer. Therefore, for SPIND-norefine and the 3-ring integration method (White et al., 2012) that is usually adopted for intensity integration using indexamajig, the background, signal and noise are incorrectly estimated since the predicted peak positions may not match the observed peak positions in the higher-resolution range without lattice refinement. The addition of an orientation refinement module requiring fewer peaks will improve merging statistics from SPIND at higher resolution than demonstrated here, if not limited by crystal quality. 


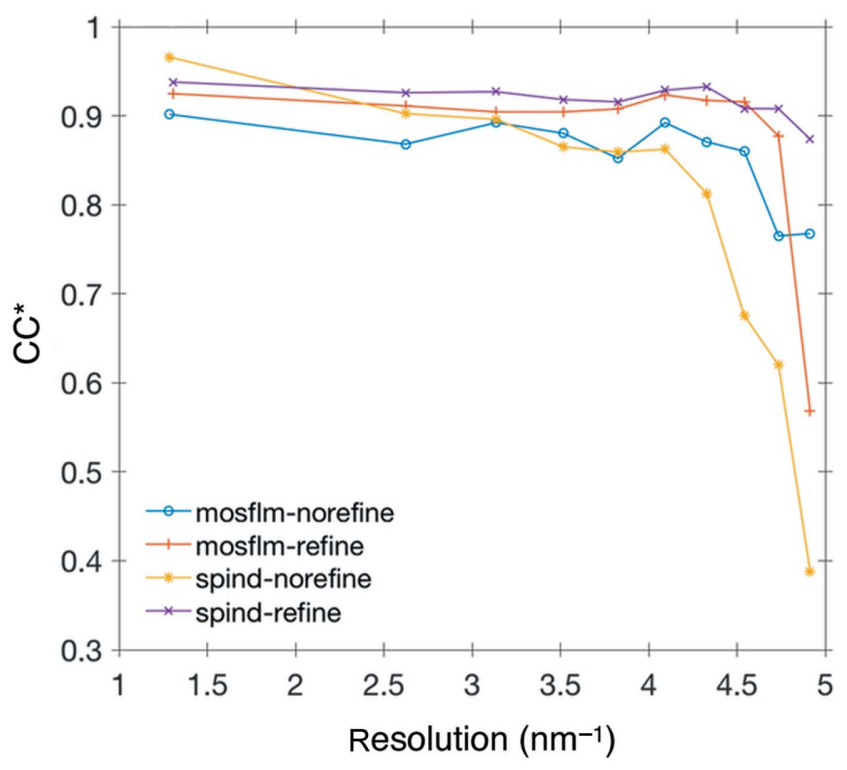

(a)

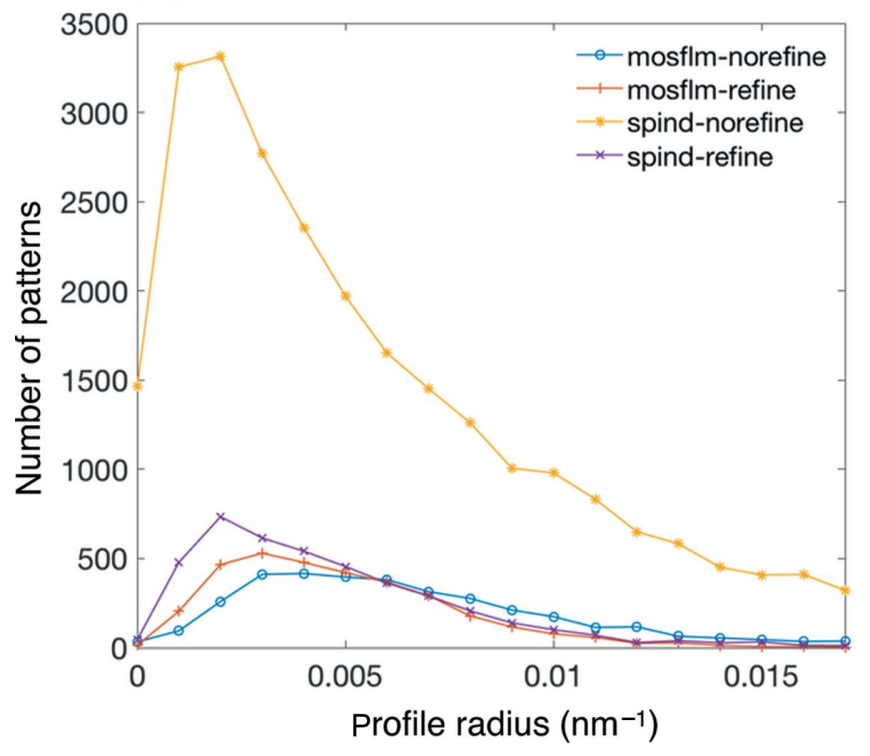

(c)

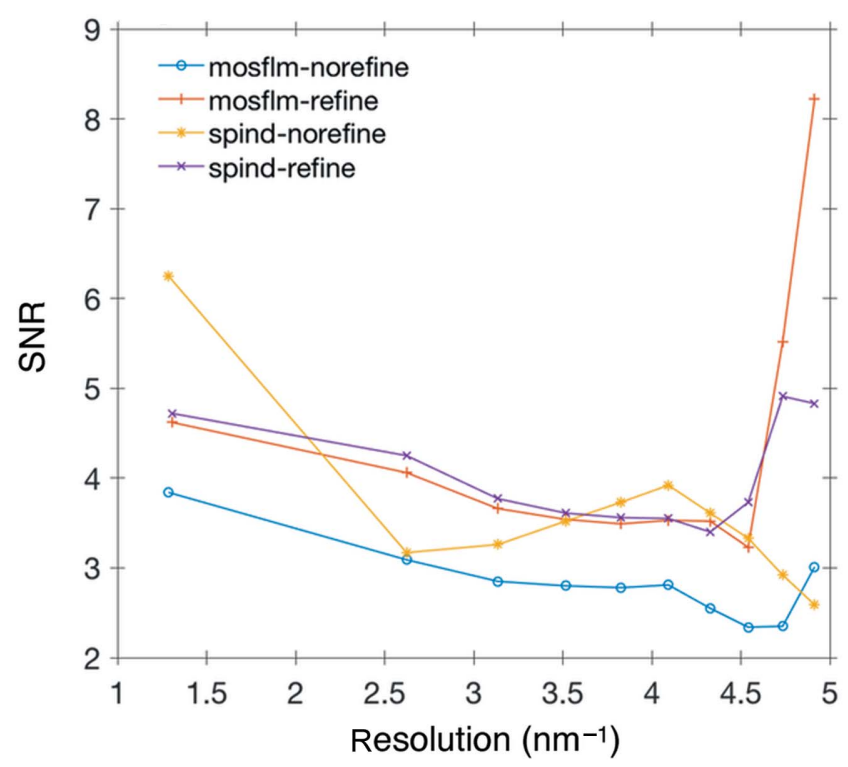

(b)

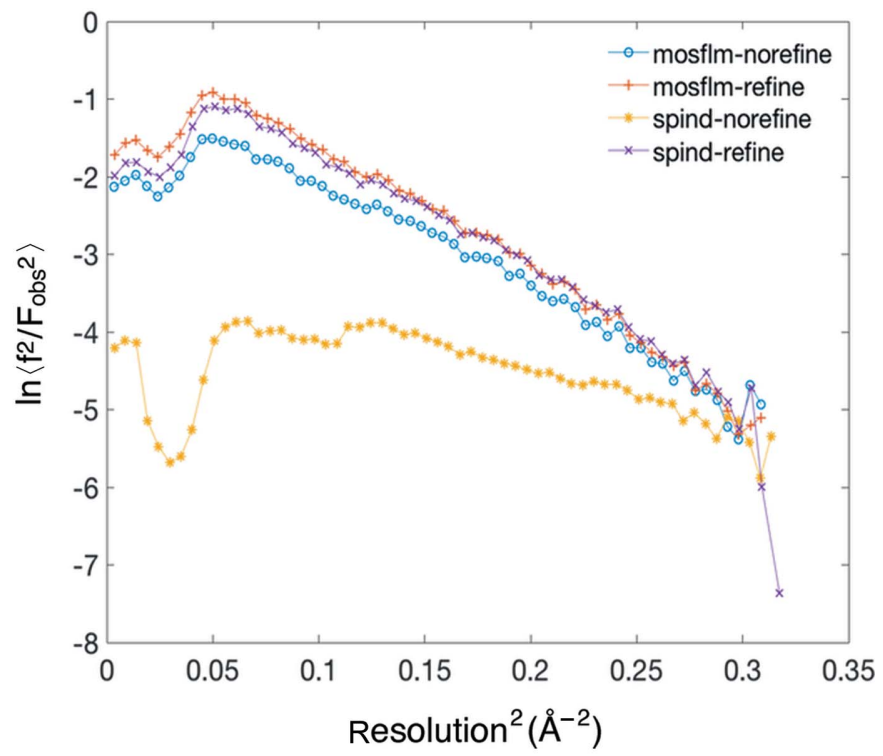

(d)

Figure 9

Figures-of-merit for the CIR data set indexed with various indexing algorithms. (a) CC*, $(b) \mathrm{SNR},(c)$ reflection profile radii and $(d)$ Wilson plots. The keywords 'refine' and 'norefine' represent the on and off status of the lattice-refinement option in indexamajig of CrystFEL in the indexing process. $S P I N D$-refine has better figures of merit for this data set than the other methods. Small modal reflection profile radii indicate that orientation determined by SPIND is often more accurate than MOSFLM with and without orientation refinement.

\subsection{Software availability, usage and performance}

The algorithm development and prototype test of SPIND were first conducted in MATLAB. For compatibility and portability, $S P I N D$ includes recently updated features designed for protein serial crystallography and is publicly available under the GNU General Public License from https:// github.com/LiuLab-CSRC/SPIND. For the user's convenience, CrystFEL, with SPIND integrated as an alternative indexing module callable from indexamajig, is also available from the repository. The CrystFEL data-analysis pipeline incorporating SPIND is shown in Fig. 11. It is recommended to use MOSFLM and DirAx etc. for auto-indexing first and then apply SPIND to improve the indexing rate based on the reference unit cell given by previous indexers.

The computation time and required memory are mainly determined by two factors. (1) the length of the structurefactor list used to generate the reference table. The computation time and required memory roughly follows $N^{2}$, where $N$ denotes the number of Bragg reflections included in the reference structure-factor list. (2) Error-tolerance threshold in the vector-searching process (see the Methods section). Larger threshold values generally lead to longer computation time. Auto-indexing using SPIND can be very time and memory demanding for protein crystallography because of the large number of Bragg reflections used in the reference list. In 

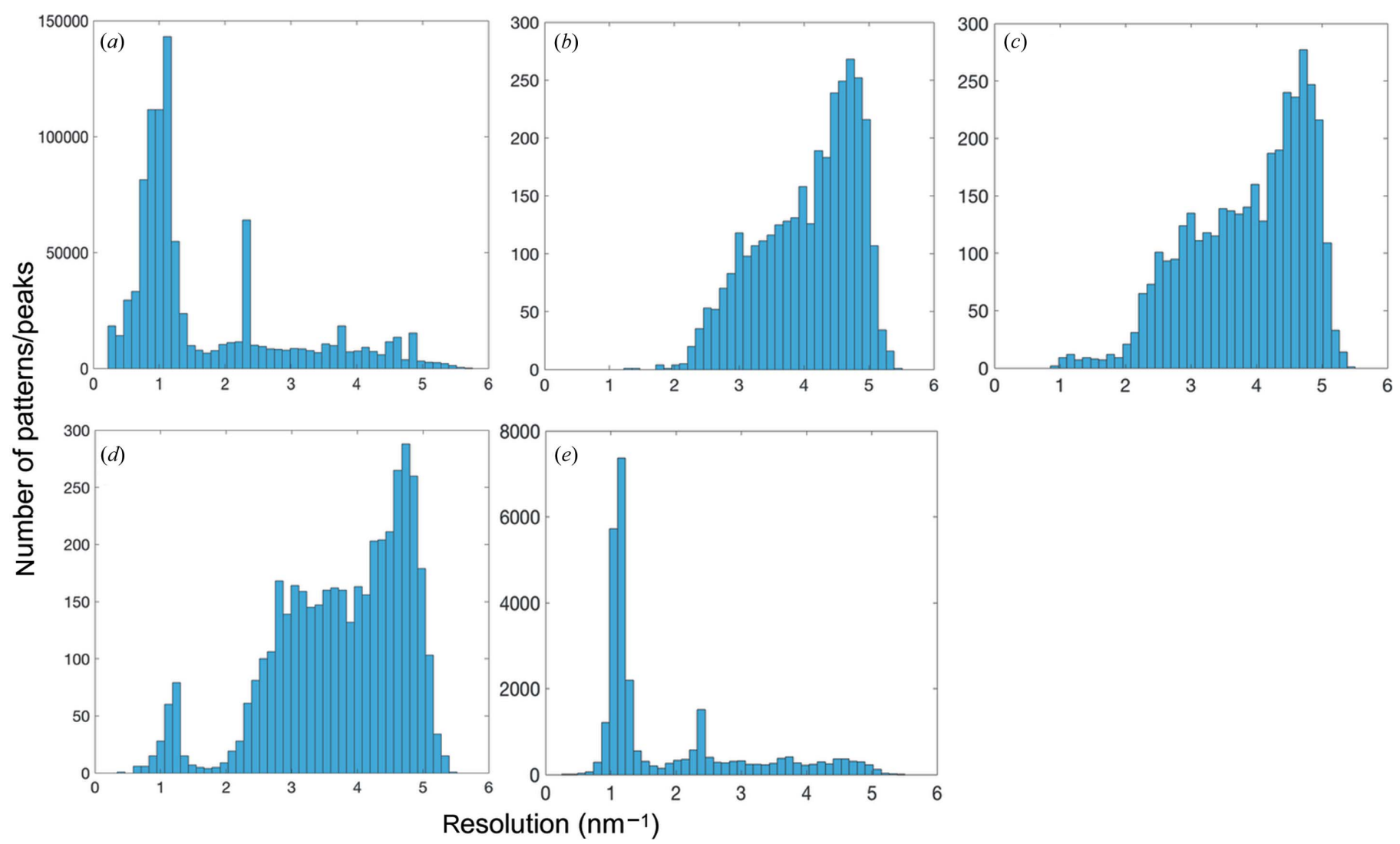

Figure 10

Resolution histograms for the ClR data set. (a) Resolution distribution of found peaks for all crystal hits, and distributions of apparent diffraction resolution determined by indexamajig after indexing by (b) MOSFLM-refine, (c) MOSFLM-norefine, (d) SPIND-refine and (e) SPIND-norefine. The additional patterns indexed by SPIND are mostly in the lower-resolution region (around $1 \mathrm{~nm}^{-1}$ ) which is consistent with the resolution distribution of the found peaks.

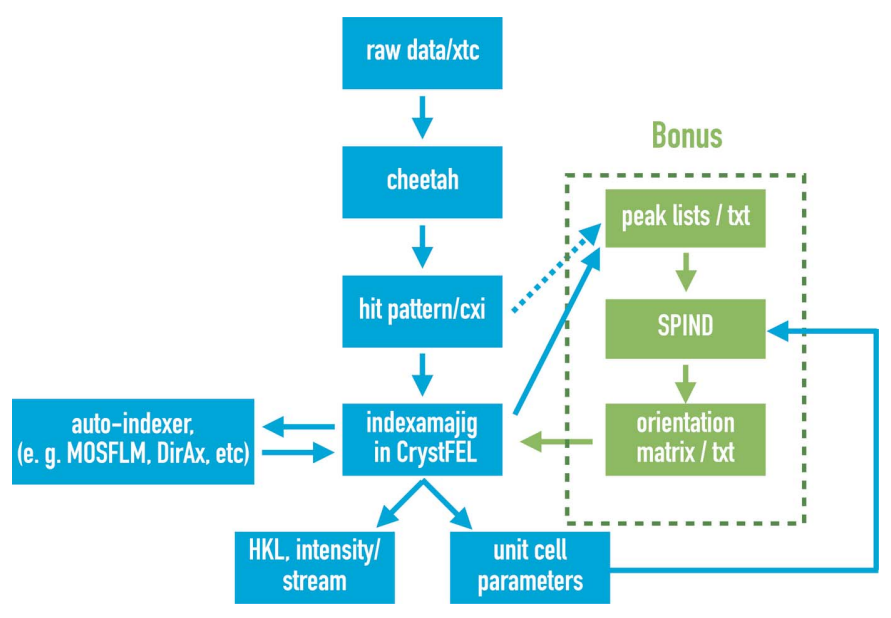

Figure 11

Schematic SFX data-analysis pipeline integrating SPIND to CrystFEL.

principle, the reference structure-factor list is generated only for the resolution range where most of the experimental peaks fall, to minimize the memory needs and computation time. In addition, the vector-tolerance threshold can be set to be small first, and then be adjusted to be larger to increase the indexing rate with a longer but reasonable computation time. SPIND also supports parallel computation using multiple CPUs. As an example, the computation time to auto-index a subset of 4962 patterns from the DOR SFX data set was around 12 core hours (Intel Xeon E5-2680 v3 at $2.5 \mathrm{GHz}$ ).

\section{Conclusions}

Diffraction patterns that consist of a small number of peaks often take up a significant portion of the whole data set in serial protein crystallography. The insufficient number of peaks along with the poor diffraction quality make these patterns difficult to analyze using the Fourier transform-based algorithms. In order to utilize these data and increase the data efficiency, we have developed a new auto-indexing algorithm, SPIND. It is based on identifying the Miller indices of five peaks chosen from each pattern by comparison with the reference unit cell.

The algorithm was tested first using simulated diffraction data from I3C microcrystals in random orientations and with random fluctuations in lattice constants. All 400 simulated sparse I3C patterns were auto-indexed successfully using only three to five Bragg peaks per pattern. Each pattern was autoindexed in milliseconds, to an accuracy of $0.1^{\circ}$ in the Euler angle defining the crystal orientation. This shows the robustness of the algorithm to lattice inhomogeneity and distortions. SPIND was then shown to perform as well as established auto- 
indexers MOSFLM and DirAx, slightly improving the indexing of SFX data from microcrystals of a GPCR complex (CXIDB ID 40). The SNR and self-consistency figures of merit all slightly improved over the whole resolution range by using SPIND with orientation refinement in indexamajig. Finally, SPIND was used to improve a data set from CIR crystals. The indexing rate using MOSFLM was around $3 \%$ because of the insufficient number of peaks in most of the patterns, along with poor diffraction quality. Even without orientation refinement in indexamajig (which requires at least ten Bragg spots), SPIND improved the merged data quality in the lower resolution range by indexing additional patterns of low resolution. However, the overall resolution limit of the whole data set was ultimately limited by the low diffraction quality of the crystals.

These results demonstrate that SPIND can index serial microcrystal diffraction patterns with very few Bragg reflections (e.g. inorganic microcrystals with small unit cells), and improve the quality of membrane-protein SFX data. The growing adoption of serial crystallography methods at synchrotron beamlines, using continuous injection of a stream of microcrystals across the beam and fast recording (Standfuss \& Spence, 2017), as well as micro-electron diffraction from inorganic and macromolecular microcrystals, will also benefit from the algorithm described here. SPIND is actively being developed to include an optimized search algorithm, multicrystal indexing, orientation refinement and more features for improving protein SFX indexing. It is written in Python and is publicly available from https:/github.com/LiuLab-CSRC/ SPIND.

\section{Acknowledgements}

We thank the group of Weontae Lee and Jihye Yun at the Department of Biochemistry in Yonsei University, Seoul, for providing the CIR SFX data set for this study, collected at the LCLS, SLAC National Accelerator Laboratory.

\section{Funding information}

This work is supported by the STC Program of the National Science Foundation through BioXFEL under Agreement No. 1231306, ABI Innovation: New Algorithms for Biological X-ray Free Electron Laser Data under NSF grant No. 1565180, and the National Natural Science Foundation of China (awarded to H. Liu) \#11575021, U1530401, and U1430237. Use of the LCLS at the SLAC National Accelerator Laboratory is supported by the US Department of Energy, Office of Science, Office of Basic Energy Sciences under contract No. DE-AC02-76SF00515.

\section{References}

Barty, A., Kirian, R. A., Maia, F. R. N. C., Hantke, M., Yoon, C. H., White, T. A. \& Chapman, H. (2014). J. Appl. Cryst. 47, 1118-1131. Beck, T. \& Sheldrick, G. M. (2008). Acta Cryst. E64, o1286.

Beyerlein, K. R., White, T. A., Yefanov, O., Gati, C., Kazantsev, I. G., Nielsen, N. F.-G., Larsen, P. M., Chapman, H. N. \& Schmidt, S. (2017). J. Appl. Cryst. 50, 1075-1083.
Brehm, W. \& Diederichs, K. (2014). Acta Cryst. D70, 101-109.

Brewster, A. S., Sawaya, M. R., Rodriguez, J., Hattne, J., Echols, N., McFarlane, H. T., Cascio, D., Adams, P. D., Eisenberg, D. S. \& Sauter, N. K. (2015). Acta Cryst. D71, 357-366.

Campbell, J. W. (1998). J. Appl. Cryst. 31, 407-413.

Cazals, F. \& Karande, C. (2008). Theor. Comput. Sci. 407, 564-568.

Chapman, H. N., Fromme, P., Barty, A., White, T., Kirian, R. A., Aquila, A., Hunter, M. S., Schulz, J., DePonte, D. P., Weierstall, U., Doak, R. B., Maia, F. R. N. C., Martin, A. V., Schlichting, I., Lomb, L., Coppola, N., Shoeman, R. L., Epp, S. W., Hartmann, R., Rolles, D., Rudenko, A., Foucar, L., Kimmel, N., Weidenspointner, G., Holl, P., Liang, M., Barthelmess, M., Caleman, C., Boutet, S., Bogan, M. J., Krzywinski, J., Bostedt, C., Bajt, S., Gumprecht, L., Rudek, B., Erk, B., Schmidt, C., Hömke, A., Reich, C., Pietschner, D., Strüder, L., Hauser, G., Gorke, H., Ullrich, J., Herrmann, S., Schaller, G., Schopper, F., Soltau, H., Kühnel, K., Messerschmidt, M., Bozek, J. D., Hau-Riege, S. P., Frank, M., Hampton, C. Y., Sierra, R. G., Starodub, D., Williams, G. J., Hajdu, J., Timneanu, N., Seibert, M. M., Andreasson, J., Rocker, A., Jönsson, O., Svenda, M., Stern, S., Nass, K., Andritschke, R., Schröter, C., Krasniqi, F., Bott, M., Schmidt, K. E., Wang, X., Grotjohann, I., Holton, J. M., Barends, T. R. M., Neutze, R., Marchesini, S., Fromme, R., Schorb, S., Rupp, D., Adolph, M., Gorkhover, T., Andersson, I., Hirsemann, H., Potdevin, G., Graafsma, H., Nilsson, B. \& Spence, J. C. H. (2011). Nature, 470, 73-77.

Duisenberg, A. J. M. (1992). J. Appl. Cryst. 25, 92-96.

Fenalti, G., Zatsepin, N. A., Betti, C., Giguere, P., Han, G. W., Ishchenko, A., Liu, W., Guillemyn, K., Zhang, H., James, D., Wang, D., Weierstall, U., Spence, J. C. H., Boutet, S., Messerschmidt, M., Williams, G. J., Gati, C., Yefanov, O. M., White, T. A., Oberthuer, D., Metz, M., Yoon, C. H., Barty, A., Chapman, H. N., Basu, S., Coe, J., Conrad, C. E., Fromme, R., Fromme, P., Tourwé, D., Schiller, P. W., Roth, B. L., Ballet, S., Katritch, V., Stevens, R. C. \& Cherezov, V. (2015). Nat. Struct. Mol. Biol. 22, 265-268.

French, S. \& Wilson, K. (1978). Acta Cryst. A34, 517-525.

Ginn, H. M., Brewster, A. S., Hattne, J., Evans, G., Wagner, A., Grimes, J. M., Sauter, N. K., Sutton, G. \& Stuart, D. I. (2015). Acta Cryst. D71, 1400-1410.

Ginn, H. M., Roedig, P., Kuo, A., Evans, G., Sauter, N. K., Ernst, O. P., Meents, A., Mueller-Werkmeister, H., Miller, R. J. D. \& Stuart, D. I. (2016). Acta Cryst. D72, 956-965.

Hart, P., Boutet, S., Carini, G., Dubrovin, M., Duda, B., Fritz, D., Haller, G., Herbst, R., Herrmann, S., Kenney, C., Kurita, N., Lemke, H., Messerschmidt, M., Nordby, M., Pines, J., Schafer, D., Swift, M., Weaver, M., Williams, G., Zhu, D., Van Bakel, N. \& Morse, J. (2012). Proc. SPIE, 8504, 85040C.

Hattne, J., Echols, N., Tran, R., Kern, J., Gildea, R. J., Brewster, A. S., Alonso-Mori, R., Glöckner, C., Hellmich, J., Laksmono, H., Sierra, R. G., Lassalle-Kaiser, B., Lampe, A., Han, G., Gul, S., DiFiore, D., Milathianaki, D., Fry, A. R., Miahnahri, A., White, W. E., Schafer, D. W., Seibert, M. M., Koglin, J. E., Sokaras, D., Weng, T.-C., Sellberg, J., Latimer, M. J., Glatzel, P., Zwart, P. H., GrosseKunstleve, R. W., Bogan, M. J., Messerschmidt, M., Williams, G. J., Boutet, S., Messinger, J., Zouni, A., Yano, J., Bergmann, U., Yachandra, V. K., Adams, P. D. \& Sauter, N. K. (2014). Nat. Methods, 11, 545-548.

Herrmann, S., Boutet, S., Duda, B., Fritz, D., Haller, G., Hart, P., Herbst, R., Kenney, C., Lemke, H., Messerschmidt, M., Pines, J., Robert, A., Sikorski, M. \& Williams, G. (2013). Nucl. Instrum. Methods Phys. Res. A, 718, 550-553.

Kabsch, W. (1988). J. Appl. Cryst. 21, 67-72.

Kabsch, W. (1993). J. Appl. Cryst. 26, 795-800.

Karplus, P. A. \& Diederichs, K. (2012). Science, 336, 10301033.

Kirian, R. A., Wang, X., Weierstall, U., Schmidt, K. E., Spence, J. C. H., Hunter, M., Fromme, P., White, T., Chapman, H. N. \& Holton, J. (2010). Opt. Express, 18, 5713-5723.

Leslie, A. G. W. (2006). Acta Cryst. D62, 48-57. 
Li, C., Schmidt, K. \& Spence, J. C. (2015). Struct. Dyn. 2, 041714.

Liang, M., Williams, G. J., Messerschmidt, M., Seibert, M. M., Montanez, P. A., Hayes, M., Milathianaki, D., Aquila, A., Hunter, M. S., Koglin, J. E., Schafer, D. W., Guillet, S., Busse, A., Bergan, R., Olson, W., Fox, K., Stewart, N., Curtis, R., Miahnahri, A. A. \& Boutet, S. (2015). J. Synchrotron Rad. 22, 514-519.

Liu, H. \& Spence, J. C. H. (2014). IUCrJ, 1, 393-401.

Liu, H. \& Spence, J. C. H. (2016). Quant. Biol. 4, 159-176.

Maia, F. R. N. C. (2012). Nat. Methods, 9, 854-855.

Maia, F. R. N. C., Yang, C. \& Marchesini, S. (2011). Ultramicroscopy, 111, 807-811.

Neutze, R., Wouts, R., van der Spoel, D., Weckert, E. \& Hajdu, J. (2000). Nature, 406, 752-757.

Nogly, P., Panneels, V., Nelson, G., Gati, C., Kimura, T., Milne, C., Milathianaki, D., Kubo, M., Wu, W., Conrad, C., Coe, J., Bean, R., Zhao, Y., Båth, P., Dods, R., Harimoorthy, R., Beyerlein, K. R., Rheinberger, J., James, D., DePonte, D., Li, C., Sala, L., Williams, G. J., Hunter, M. S., Koglin, J. E., Berntsen, P., Nango, E., Iwata, S., Chapman, H. N., Fromme, P., Frank, M., Abela, R., Boutet, S., Barty, A., White, T. A., Weierstall, U., Spence, J., Neutze, R., Schertler, G. \& Standfuss, J. (2016). Nat. Commun. 7, 12314.

Powell, H. R. (1999). Acta Cryst. D55, 1690-1695.

Sauter, N. K. (2015). J. Synchrotron Rad. 22, 239-248.
Sauter, N. K., Grosse-Kunstleve, R. W. \& Adams, P. D. (2004). J. Appl. Cryst. 37, 399-409.

Schmidt, M. (2013). Adv. Condens. Matter Phys. 2013167276.

Spence, J. C. H. (2017). IUCrJ, 4, 322-339.

Standfuss, J. \& Spence, J. (2017). IUCrJ, 4, 100-101.

Steller, I., Bolotovsky, R. \& Rossmann, M. G. (1997). J. Appl. Cryst. 30, 1036-1040.

Uervirojnangkoorn, M., Zeldin, O. B., Lyubimov, A. Y., Hattne, J., Brewster, A. S., Sauter, N. K., Brunger, A. T. \& Weis, W. I. (2015). eLife, 4, e05421

White, T. (2014). Philos. Trans. R. Soc. B Biol. Sci. 369, 20130330.

White, T. A., Barty, A., Liu, W., Ishchenko, A., Zhang, H., Gati, C., Zatsepin, N. A., Basu, S., Oberthür, D., Metz, M., Beyerlein, K. R., Yoon, C. H., Yefanov, O. M., James, D., Wang, D., Messerschmidt, M., Koglin, J. E., Boutet, S., Weierstall, U. \& Cherezov, V. (2016). Sci. Data, 3, 160057.

White, T. A., Kirian, R. A., Martin, A. V., Aquila, A., Nass, K., Barty, A. \& Chapman, H. N. (2012). J. Appl. Cryst. 45, 335-341.

White, T. A., Mariani, V., Brehm, W., Yefanov, O., Barty, A., Beyerlein, K. R., Chervinskii, F., Galli, L., Gati, C., Nakane, T., Tolstikova, A., Yamashita, K., Yoon, C. H., Diederichs, K. \& Chapman, H. N. (2016). J. Appl. Cryst. 49, 680-689. 